
\title{
28 Research Square \\ Multiscale imaging of the rat brain using an integrated diceCT and histology workflow
}

Paul M. Gignac

Oklahoma State University Center for Health Sciences

Haley D. O'Brien

Oklahoma State University Center for Health Sciences

Jimena Sanchez

Universidad Veracruzana

Dolores Vazquez Sanroman ( $\nabla$ dolores.vazquez_sanroman@okstate.edu )

Oklahoma State University Center for Health Sciences

\section{Research Article}

Keywords: immunofluorescence, fluorescence, cresyl violet, iodine, eosinophilic, isoelectric

Posted Date: February 19th, 2021

DOI: https://doi.org/10.21203/rs.3.rs-158407/v1

License: (9) This work is licensed under a Creative Commons Attribution 4.0 International License. Read Full License 


\section{Abstract}

Advancements in tissue visualization techniques have spurred significant gains in the biomedical sciences by enabling researchers to integrate their datasets across anatomical scales. Of particular import are techniques that enable the interpolation of multiple hierarchical scales in samples taken from the same individuals. This study demonstrates that two-dimensional histology techniques can be employed on neural tissues following three-dimensional diffusible iodine-based contrast-enhanced computed tomography (diceCT) without causing tissue degradation. This represents the first step toward a multiscale pipeline for brain visualization. We studied brains from adolescent male Sprague-Dawley rats, comparing experimental (diceCT-stained then de-stained) to control (without diceCT) brains to evaluate neural tissues for immunolabeling integrity, compare somata sizes, and distinguish neurons from glial cells within the telencephalon and diencephalon. We hypothesized that if experimental and control samples do not differ significantly in quantitative metrics, brain tissues are robust to the chemical, temperature, and radiation environments required for these multiple, successive imaging protocols. Visualizations for experimental brains were first captured via micro-computed tomography scanning of isolated, iodine-infused specimens. Samples were then cleared of iodine, serially sectioned, and prepared again using immunofluorescent, fluorescent, and cresyl violet labeling, followed by imaging with confocal and light microscopy, respectively. Our results show that many neural targets are resilient to diceCT imaging and compatible with downstream histological staining as part of a low-cost, multiscale brain imaging pipeline.

\section{Introduction}

Scientific gains in understanding biological organization and complexity are closely associated with technological and methodological gains in bio-imaging. Visualization tools widely used in biomedical and clinical imaging, such as light-sheet microscopy (Voie et al. 1993), magnetic resonance imaging (MRI: Jackson and Langham 1968; Lauterbur 1974; Damadian et al. 1976; Henshaw et al. 1977; $\mu$ MRI: Smith et al. 1996; Dodd et al. 1999), white matter tractography (diffusion tensor image analysis; Basser et al. 1994; Jellison et al. 2004), X-ray micro-computed tomography ( $\mu$ CT; Hounsfield 1977; Feldkamp et al. 1989; Rüegsegger et al. 1996), and positron emission tomography (Ter-Pogossian et al. 1975; Phelps et al. 1976; Muehllehner et al. 1976; Hawkins et al. 1992; Jones and Townsend 2017), are continuously refined to allow investigators to improve the visualization of gross anatomical, microscopic, and cellularlevel structure and function of biological tissues. These methodological enhancements have spurred significant advancement within the biomedical sciences by enabling researchers to integrate datasets across anatomical scales--from the low-resolution capture of gross structures to the fine-resolution capture of subcellular cytoskeletal elements. Synthetic approaches that combine multiple imaging techniques have the potential to maximize single-specimen data extraction that spans hierarchical, logarithmic scales of tissue organization.

Here, we evaluate the efficacy of using diffusible iodine-based contrast-enhanced computed tomography (diceCT; Gignac et al. 2016) as a low-cost foundation for multiscale anatomical imaging integration by 
combining high-resolution cellular and gross anatomical brain-visualization techniques. Although samebrain, multiscale visualization has been conducted previously by combining MRI with histology (e.g., Ding et al. 2016), the relatively coarse resolution of most readily-available MRI equipment limits the spatial accuracy of placing microscopic-scale information from histology into macro- and mesoscopic anatomical contexts. $\mu \mathrm{MRI}$ can achieve more satisfactory spatial resolutions as high as 25 microns, potentially overcoming this limitation; however, $\mu \mathrm{MRI}$ scans remain cost-prohibitive, time-consuming, and are hampered by specimen size limitations (de Crespigny et al. 2008). Nonetheless, $\mu M R I$ remains a popular imaging modality because it is non-disruptive to biological macromolecules, including proteins (Smith et al. 1996). We propose the use of diceCT (Gignac et al. 2016), which is commonly employed in organismal biology research, as a novel, inexpensive, and high-resolution ex vivo alternative to $\mu \mathrm{MRI}$. DiceCT is an X-ray $\mu \mathrm{CT}$-imaging technique that utilizes iodine (in the form of Lugol's iodine $\left[\mathrm{I}_{2} \mathrm{KI}\right]$ or alcoholic iodine $\left[\mathrm{I}_{2} \mathrm{E}\right]$ ) as a contrast agent to permit three-dimensional (3D) visualization of tissue-level neuroanatomy at resolutions superior to MRI-based modalities (de Crespigny et al. 2008; Anderson and Maga 2015; Hughes et al. 2016b; Gignac and Kley 2018). lodine absorbs X-rays and binds preferentially to sugars and fatty tissues, making it excellent for nervous system visualization due to its clear differentiation of myelinated and non-myelinated structures at micron-scale resolution using $\mu \mathrm{CT}$ (Gignac and Kley 2018).

The application of $\mu \mathrm{CT}$ for studying how the brain is organized has lagged behind its use as a diagnostic tool in biomedical studies (Sengle et al., 2013; Hopkins et al., 2015; Karreman et al. 2016; Morales et al. 2016; Shami et al. 2017). However, $\mu$ CT-scanner technologies and software optimizations are improving rapidly, allowing increasingly faster scans at micro-and nano-scale resolutions (e.g., Pelc 2015). The swift evolution of $\mu \mathrm{CT}$ imaging is now being leveraged to document a broad array of tissue types at various spatial scales, thereby providing a substrate for multi-scale visualization as, or perhaps more, viable than $\mu \mathrm{MRI}$. For example, diceCT methods achieve simultaneous visualization of hard and soft tissues, such as the skull with its brain and cranial nerves intact (Gignac and Kley, 2018). They also include reversible processing steps, allowing multiscale, fully-registered imaging of the same tissues with more traditional histological approaches. Multi-tool and correlative imaging, such as that widely used in pathology for the structural examination of human tissues (McDonald and Hayes 1969; Morales et al. 2016), enables multiscale co-registration datasets from the same sample. Multiscale imaging via diceCT, therefore, holds the potential to maximize the amount of neuroanatomical information retrieved from one individual, which is otherwise practically unattainable using single-scale and destructive approaches (Caplan et al. 2011; Burnett et al. 2014).

Recently, diceCT samples have been used successfully in subsequent thin-section histological protocols. These studies have followed a variety of methodologies, including harnessing the iodine already present in the sample from initial contrast-enhancement (Jeffery et al. 2011) or following a dehydration rinse in ethanol with re-staining using either Giemsa (Herdina et al. 2015), thionin (Hughes et al. 2016a), hematoxylin and eosin (Chen et al. 2018), Marius yellow, crystal red, methyl blue, and Luxol fast blue solutions (Heimel et al. 2019). Histological results secondary to diceCT appear to have produced clear 
identification of two-dimensional (2D) muscular, epithelial, connective, and peripheral nervous tissues. Collectively, these studies illustrate the potential for multiscale diceCT-to-histology tissue-imaging pipelines. However, no studies have formally tested post-diceCT histology against non-diceCT controls, nor have any reported successful immunocytochemical and immunofluorescence tissue staining following diceCT.

Brain tissue is considered to be incredibly delicate, with neural proteins highly susceptible to degradation under non-idealized chemical and/or thermal conditions (Gwóźdź et al. 1970; Bowler and Tirri 1974; Gwóźdź et al. 1978; Millan et al. 1979; Gold et al. 1985) when compared to somatic tissues (Burger and Fuhrman 1964). Destructive ex vivo conditions may include formalin fixation, weeks in a salt-rich and aqueous contrast solution (e.g., $\mathrm{I}_{2} \mathrm{KI}$ ), repeated staining with refreshed solutions, storage at room temperature, exposure to high levels of X-rays, and de-staining with alternating baths of sodium thiosulfate $\left(\mathrm{Na}_{2} \mathrm{~S}_{2} \mathrm{O}_{3}\right)$ and deionized water or $70 \%$ ethanol-each of which represents a step in diceCT preparation, imaging, and de-staining protocols (Gignac et al. 2016). The long-term resiliency of nervous tissues to such conditions has not been quantified. To directly address the susceptibility of neurons and glial cells to degradation during diceCT preparation and imaging, we compare diceCT treatment and control neural tissue samples by: 1) evaluating the specificity and integrity of fluorescence, immunofluorescence, and cresyl violet staining methods; 2) analyzing preserved morphologies; and 3) quantifying neural cells in four regions of interest within the telencephalon and diencephalon. We hypothesize that, given the proper conditions, brain tissue can remain robust to the chemical, temperature, and radiation environments required for multiple imaging protocols, and we quantitatively evaluated this hypothesis by comparing diceCT-histology preparations and histology-only controls. To help guide future studies, we also document instances in our evaluation of these protocols wherein deviating from certain steps in the process could preclude successful histological staining. Overall, we demonstrate that diceCT is non-destructive of neural tissues, and our results support the use of diceCT imaging in lieu of $\mu \mathrm{MRI}$ as part of a multiscale brain imaging pipeline.

\section{Methods}

\subsection{Brain sampling}

Six adolescent (120-150 g body weight) male Sprague-Dawley rats (Charles River Laboratories, Franklin, MA, USA) were single-housed on a 12/12-hour light/dark cycle with ad libitum access to food and water. All rats were deeply anesthetized with an Avertin/xylazine cocktail and perfused transcardially with cold saline solution $(0.9 \% \mathrm{NaCl})$ followed by cold $4 \%$ paraformaldehyde. After perfusion, cervical vertebrae were removed, followed by postcranial musculature and surrounding connective tissue. Dorsal portions of the parietal and temporal skull bones were detached, and the entire occipital bone was excised. The remaining tissues connecting the skull and brain were severed, and all cranial nerves cut at the roots. Once extracted, brains were placed in $4 \%$ paraformaldehyde solution and refrigerated $\left(4^{\circ} \mathrm{C}\right)$ for 24 hours, followed by a $0.1 \mathrm{M}$ Phosphate Buffer Solution (PBS-30\%) with refrigeration $\left(4^{\circ} \mathrm{C}\right)$ for $48-96$ hours, at 
which full point immersion was observed. Three brain samples were prepared for diceCT imaging, followed by cresyl violet, fluorescence, and immunofluorescence staining. Three additional brain samples were prepared as histological controls.

\subsection{DiceCT protocols \\ 2.2.1 Staining}

Following 24 hours of fixation and up to 96 hours of sucrose immersion, experimental rat brains $(n=3)$ were submerged into a $3 \%$ weight-by-volume $(\mathrm{w} / \mathrm{v})$ solution of Lugol's iodine $\left(\mathrm{I}_{2} \mathrm{KI}\right)$ for 21 days (at room temperature), following protocols outlined in Gignac et al. (2016) and Gignac and Kley (2018). In an aqueous solution, iodine $\left(\mathrm{I}_{2}\right)$ and potassium iodide $(\mathrm{KI})$ become Triiodide (iodine trimers; $\mathrm{I}_{3}{ }^{-}$) and potassium ions $\left(\mathrm{K}^{+}\right)$, and iodine trimers bind differentially to the carbohydrates and lipids present in neural tissues. All three samples were fully submerged in an iodine solution, placed on an electric rocker, and protected from light for stain infusion duration. Upon completion, specimens were washed in a deionized water bath for two hours to remove unbound, excess staining agent from external surfaces, which further aids in the differentiation of neural tissues during X-ray imaging (Gignac and Kley 2018). Specimens were then sealed in $50 \mathrm{ml}$ polypropylene centrifuge tubes with polyethylene foam and filled with deionized water for CT scanning. Foam and centrifuge tubes were chosen as packing material and containers, respectively, for their relatively low density compared to iodine-stained tissues. Samples were fully submerged in water to prevent desiccation during scanning.

\subsection{2 $\mu$ CT scanning}

All specimens were $\mu \mathrm{CT}$ scanned on a GE phoenix v|tome|x s240 high-resolution micro-focus CT system (General Electric, Fairfield, CT, USA) at the American Museum of Natural History Microscopy and Imaging Facility (New York, NY, USA). Scan parameters were optimized on a specimen-by-specimen basis, guided by the scout $X$-ray image and accompanying histogram. All scan parameters follow the recommendations of Gignac and Kley (2018). Due to the similarity of each specimen's size, shape, tissue constituency, and staining, all scans were performed within a narrow range of 110-120 kilovolts, 110120 micro-Amperage, 333-millisecond exposure timing, and using six $\times$ multi-frame averaging on a tungsten target with a $0.1 \mathrm{~mm}$ copper filter. All scans were completed in 107 minutes at 20-25 micrometer $(\mu \mathrm{m})$ resolutions (isometric voxels) to demonstrate equivalency to the highest $\mu \mathrm{MRI}$ resolutions currently available. After scanning, $\mu \mathrm{CT}$ data were reconstructed on an HP z800 workstation (Hewlett-Packard, Palo Alto, CA, USA) equipped with VG Studio Max (Volume Graphics GmbH, Heidelberg, Germany) and exported as TIFF stacks. Stacks were imported into AvizoLite 2020 (Thermo Fisher Scientific Inc., Waltham, MA) for 3D model building based on contrast differences between white and gray matter structures, as described in Gignac and Kley (2018).

\subsubsection{Chemical de-staining}

Following X-ray imaging, we used a chemical de-staining process to remove iodine from brain tissues. Brains were sectioned transversely into anterior and posterior halves (parallel to the planned plane of 
histological section) to increase the surface area available for the de-staining agent's diffusion. Our team's preliminary efforts identified this bisection of the brains into anterior-posterior halves as a vital step to ensure complete removal of the triiodide contrast agent. Without this step, subsequent histological sectioning resulted in shearing, which left the tissues unsuitable for further histological processing. All specimens were chemically de-stained by submersion in a $1 \% \mathrm{w} / \mathrm{v}$ solution of sodium thiosulfate on an electric rocker for seven days, followed by three days of a deionized water bath to remove excess de-staining agent (Gignac et al. 2016). In solution, sodium thiosulfate reacts with Triiodide, reducing it to iodide $\left(\mathrm{I}^{-}\right)$and chemically de-staining soft tissues (Trevorrow and Fashena 1935; Shakhashiri 1983; Kondo et al. 2001). This protocol was undertaken twice in total. Repetition ensured that all iodine was chemically unbound from and physically flushed out of samples before histological sectioning and re-staining. We recommend visual inspection of tissue slides at the beginning of histological preparation to confirm that samples are de-stained and free of any residual brown to orange coloration.

\subsection{Histology preparation}

Histological control specimens $(n=3)$ were treated following protocols previously published (VazquezSanroman et al., 2017). De-stained (i.e., post-diceCT) and control brains were transferred to a PBS- $30 \%$ sucrose solution for one week. In preparation for cryo-sectioning, samples were embedded using Histoprep frozen tissue embedding medium (Thermo Fisher Scientific Inc.) and immersed in liquid nitrogen for 20 seconds. Consecutive coronal sections, $15 \mu \mathrm{m}$ in thickness, were obtained using a cryostat on a $-6^{\circ} \mathrm{C}$ setup (Ag Protect Leica CM 1860; Leica Camera AG, Wetzlar, Germany) and stored in an antifreeze solution ( $30 \%$ ethylene glycol, $25 \%$ glycerol, $30 \%$ sucrose in PBS) at $-20^{\circ} \mathrm{C}$. Histoprep and liquid nitrogen immersion were critical for optimizing tissue sectioning protocol. Besides, sections thicker than $15 \mu \mathrm{m}$ tended to disintegrate upon handling, which left them unreliable for staining.

Areas from the telencephalon and diencephalon were pre-selected for histological analyses based on expected clear anatomical boundaries and identifiable neuronal or glial cell populations. We probed a variety of brain regions to select cell-type-specific markers that distinguish neurons and glial cells. We also tested for differences between control and post-diceCT preparations to determine if diceCT interferes with immunofluorescence, fluorescence, and standard histological stains commonly used to identify neural phenotypes. We used five stains: three immunofluorescent labels (anti-parvalbumin [PRV], antiglial fibrillary antigen protein [GFAP], and anti-DARPP-32), one fluorescent label for DNA (4',6-diamidino-2phenylindole [DAPI]), as well as a cresyl violet stain for RNA. These were applied as relevant to the medial prefrontal cortex (PRV, DAPI, cresyl violet), mammillary nucleus (GFAP, DAPI), the dentate gyrus of the hippocampus (PRV, DAPI, cresyl violet), and the substantia nigra pars reticulata (anti-DARPP-32, DAPI). These targeted antibodies allowed us to characterize phenotypes for cell membrane proteins, intermediate filaments, nucleic acids, and dopamine-cAMP-regulated phosphoprotein. See Table 1 for a summary of histological preparations.

\subsubsection{Fluorescent staining}


In order to qualitatively and quantitatively examine overall cellular morphologies and to ensure the viability of tissues for antibody reactivity, tissue sections containing portions of the medial prefrontal cortex (mPFC), mammillary nucleus (MM), the dentate gyrus of the hippocampus (DG), and substantia nigra pars reticulata ( $\mathrm{SNr}$ ) from all experimental and control specimens were prepared for immunofluorescent staining. Free-floating sections were rinsed seven times for 15 minutes each at room temperature with Triton X-100:1× Tris-PBS (Tris- $\mathrm{HCl} 10 \mathrm{mM}$, sodium phosphate buffer $10 \mathrm{mM}, 0.9 \% \mathrm{NaCl}$, $\mathrm{pH}$ 7.4) and incubated at $4^{\circ} \mathrm{C}$ with the following primary antibodies: (1) mouse monoclonal antiparvalbumin (PRV) antibody (no. 235, Swant Inc., Marly, Switzerland), diluted 1:1000; (2) mouse monoclonal anti-glial fibrillary antigen protein (GFAP) (no. 3670, Cell Signaling Technology, Danvers, MA, USA), diluted 1:500; or (3) goat polyclonal anti-DARPP-32 (no. sc-271111, Santa Cruz Biotechnology, Dallas, TX, USA), diluted 1:200. Incubations were at $4^{\circ} \mathrm{C}$ for 24 hours in PBS 0.1M Triton X-100 containing 3\% donkey serum (no. sc-2044, Santa Cruz Biotechnology). After rinsing, tissue was protected from light during a two-hour incubation at room temperature with one of the following secondary antibodies with conjugated fluorochromes: (1) Alexa Fluor 488 donkey anti-mouse (no. A32766 Thermo Fisher Scientific) diluted 1:500; or (2) Alexa Fluor 488 donkey anti-goat (no. 705-545-147 Jackson Labs, Bar Harbor, ME, USA), diluted 1:500; followed by 4',6-diamidino-2-phenylindole (DAPI) staining for one minute (no. sc-3598, Santa Cruz Biotechnology). Once the fluorescence reaction occurred, sections were mounted using Mowiol 4-88 reagent (475904-100GM, MilliporeSigma, Burlington, MA, USA).

\subsubsection{Cresyl violet staining}

To test the viability of tissues and cellular morphologies for non-fluorescent labeling, cresyl violet staining was performed using the MPFC and DG sections. The number of cells was quantitively analyzed from DG-containing sections. Free-floating slices [inferred Bregma coordinates: DG: (- $5.3 \mathrm{~mm})$; mPFC: (3.20 $\mathrm{mm})$; Paxinos and Watson, 2003] were rinsed and then mounted on gelatin-coated slides. Sections were stained for eight minutes in $0.1 \%$ cresyl violet (no. 190-M, Sigma-Aldrich Corp., St. Louis, MO, USA), followed by two rinses of distilled water for 30 seconds each. Sections were subsequently dehydrated in ascending ethanol concentrations (50-100\%; one minute each) and cleared in two xylene changes. Slides were then covered with the DPX mounting medium (no. 06522; Sigma-Aldrich Corp.) and imaged at $\times 4, \times 10, \times 20$, and $\times 100$ magnifications.

\subsection{Densitometry measurements and cellular metrics}

All fluorescent-labeled sections for each region of interest (mPFC, MM, DG, SNr) were examined using confocal microscopy (Nikon C1 Digital Eclipse Modular Confocal Microscope System; Nikon Corp., Tokyo, Japan). Confocal images were taken in single XY planes at a resolution of $1024 \times 1024$ and $100 \mathrm{~Hz}$ speed. Laser intensity, gain, and offset were maintained constant for each image acquisition. Quantitative evaluations were made using ImageJ software (National Institutes of Health, Bethesda, MD, USA). For the densitometry analysis (GFAP fluorescence), images were transformed from RGB into binary (black and white) to define objects and backgrounds. ImageJ plugins for noise reduction and background subtraction were applied across all images for each condition. GABAergic neurons visualized in the postdiceCT and control tissues were confirmed by the positive staining of PRV. Immunoreactivity was 
abundant in somata, axons, terminals, and cells with the general appearance of those reported by earlier investigations, including perfused-fixed brains (Alonso et al., 1990). Parvalbumin-immunoreactive somata displayed varying morphologies and sizes. All immunoreactivity analyses were determined by measuring densitometry in $40,000 \mu \mathrm{m}^{2}$ regions of interest. Results were expressed as region-specific mean values and standard errors of the mean (SEM). Densitometry measurements were statistically compared using one-way ANOVAs. Quantitative evaluations of cellular morphologies were made using ImageJ software. Region-specific cell sizes and nuclei counts between control and post-diceCT groups were expressed as mean values with SEM and compared using one-way ANOVAs. Student's $t$-tests were employed to compare somata diameters between tissue treatments. In all statistical tests, experimental samples were compared to controls with the significance set to $\alpha<0.05$. All statistical comparisons were conducted using the STATISTICA 7 software package (Statsoft Inc., Tulsa, OK, USA).

\subsubsection{Medial prefrontal cortex: Parvalbumin, DAPI, and cresyl violet staining}

For mPFC-containing tissue sections, we quantified PRV and DAPI reactivity and compared treatments using a one-way ANOVA. Parvalbumin immunoreactive neurons in the mPFC have an identifiable structure and are well recognized as GABAergic interneurons. Therefore, they represent an excellent target phenotype to study changes in cellular morphologies. We considered PRV-GABAergic neurons to be those cells that expressed PRV in the soma and axonal projections (Alonso et al., 1990). Additionally, we estimated the number of nuclei positively stained by DAPI and assessed somatic diameter, also for oneway ANOVA. Cresyl violet was performed and compared qualitatively between post-diceCT and nondiceCT samples and with cresyl violet-stained sections from the DG.

\subsubsection{Mammillary nucleus: DAPI and glial fibrillary acidic protein}

We stained MM sections with DAPI and also labeled the sections using a GFAP antibody. We quantified the GFAP-immunoreactivity of astrocytes and compared their numbers by treatment using a one-way ANOVA and compared cell-sizes using a student's t-test.

\subsubsection{Dentate gyrus: Cresyl violet and parvalbumin staining}

For DG-containing tissue sections, cresyl violet was performed and qualitatively compared between postdiceCT and non-diceCT samples from the DG and the mPFC. Nuclei counts were also drawn from cresyl violet-stained samples and compared using one-way ANOVA. Somata sizes for PRV-positive neurons between treatments were analyzed using a student's $t$ test.

\subsubsection{Substantia nigra: DARPP-32}

For SNr sections, we quantified DARPP-32 immunoreactivity for one-way ANOVA to determine whether diceCT conditions affect intracellular messengers' quality and specificity coupled to specific receptors for 
the phosphoprotein DARPP-32, which is typically high for the SNr (Hemmings and Greengard 1986). Also, we contrasted cell sizes for DARPP-32-immunoreactive neurons in the SNr using a student's t-test.

\subsection{Digital reconstruction}

Each diceCT brain was digitally rendered into 3D, using Avizo software running on a Mac Pro computer (Apple Inc.; Cupertino, CA, USA). The slice tool was used to move through the 3D volume in planar sections to match 2D histology images with 3D diceCT anatomy. Digital images of the histology sections were virtually placed into anatomical position with their complementary diceCT volume by matching homologous neuroanatomical features. Anatomical relationships were verified against rat-brain atlases (Paxinos and Watson 2003; Swanson 2018).

\section{Results}

\subsection{DiceCT imaging}

DiceCT revealed that soft-tissue traits, even delicate myelinated structures (Gignac and Kley, 2018), could be effectively imaged and visualized (Fig. 1). Clear visualization of tissue-level structures relied on several steps to ensure imaging noise was minimized, including a post-staining wash in deionized water, moderate X-ray exposure timing during CT scanning, and several-fold multi-frame image averaging in captured X-ray image processing.

\subsection{Histological phenotypes}

\subsubsection{Medial prefrontal cortex: Parvalbumin, DAPI, and cresyl violet staining}

Parvalbumin immunoreactivity in MPFC neurons was within previously reported ranges (e.g., VazquezSanroman et al., 2017). Cells showed a morphologically heterogeneous subpopulation of non-pyramidal interneurons with PRV-positive axons and pericellular basket-like morphologies that enveloped the perikarya of nearby cell bodies (Fig. 2A, B). DiceCT staining did not alter the number of PRV-positive neurons in comparison to control brain tissue (one-way ANOVA; $\mathrm{F}_{1,10}=8.36, p=0.89$ ) (Fig. 2E,F). As an additional histological control, DAPI staining was used to determine the number of nuclei and assess gross cell morphology (Fig. 2A, B). The diceCT pipeline did not modify the appearance of cells stained with DAPI, nor surface immunostaining intensity (one-way ANOVA; $F_{1,10}=5.26, p=0.96$ ). Somata diameters in PRV-DAPI-positive cells were similar between post-diceCT and control brain tissues $(t=0.44$ $\left.\left(F_{1,14}\right), p=0.24\right)$ (Fig. $\left.2 \mathrm{G}\right)$. Brightfield light microscopy of cresyl violet-treated post-diceCT sections was sufficient to reveal cytoarchitectural landmarks (Figs. 1D, 2C). Post-diceCT cresyl violet staining quality in the MPFC was comparable to that of the post-diceCT cresyl violet-stained DG treatments (see below). 


\subsubsection{Mammillary nucleus: DAPI and glial fibrillary acidic protein}

Qualitatively, diceCT preparation, imaging, and de-staining did not modify the appearance of cells stained with DAPI. The distribution of GFAP immunoreactive elements in controls was consistent with previous reports (Rahati et al., 2016; Vazquez-Sanroman et al., 2017). Intensely stained, evenly distributed astrocytes with full dendritic body labeling characterized both post-diceCT and control tissues. However, surface densitometry of cell body and astrocyte projections revealed that diceCT-treated brain tissue decreased GFAP levels compared to controls (one-way ANOVA; $F_{1,8}=6.33, p<0.01$ ) (Fig. 3A-C).

\subsubsection{Dentate gyrus: cresyl violet and parvalbumin staining}

Brightfield light microscopy of cresyl violet-treated experimental sections revealed sufficiently robust staining to delimit specific brain regions and other cytoarchitectural landmarks in the DG. This result indicates both the successful removal of iodine and the cresyl violet dye's infiltration into the cell bodies of the treatment tissues (Fig. 4A). Cresyl violet-stained cells from post-diceCT treatments were not structurally different as compared to those from control brains. Likewise, cresyl violet staining subsequent to diceCT staining, imaging, and de-staining procedures did not alter cell nuclei counts (oneway ANOVA; $\left.F_{1,10}=18.22, p=0.66\right)$. Experimental tissues, therefore, showed preserved cell morphology, neuronal density, and tissue integrity at high-power magnification (Fig. 4A). In addition, the appearance of PRV immunoreactivity was visible in post-diceCT and control brain tissues (Fig. 4C), and neuronal somata diameters for experimental treatments did not differ from those of the controls $\left(t=1.24\left(\mathrm{~F}_{1,10}\right), p\right.$ $=0.92)($ Fig. 4C,D).

\subsubsection{Substantia nigra: DARPP-32}

We found consistent DARPP-32 expression in post-diceCT and control brains (Fig. 5B). We observed strong staining in cell bodies and projections of dopaminergic neurons within the SNr (Fig. 5B). DARPP32 co-localization with DAPI demonstrated nuclear staining in both tissue treatments. We found no significant difference in somata diameters between experimental and control brain tissues $(t=0.84$ $\left.\left(F_{1,14}\right), p=0.41\right)($ Fig. $5 C)$

\section{Discussion}

X-ray imaging of non-mineralized soft tissues is a significant challenge for neuroanatomical studies, causing the brain to be intrinsically difficult to document through such means. Contrast agents, like iodine, potentially address this issue by enabling the visualization of soft neuroanatomical features at high resolutions (Wong et al. 2013; Lautenschlager et al. 2014; Gignac et al. 2016; Parlanti et al., 2017; Naumann and Olsson 2018). However, neural tissues' resilience to multi-faceted imaging pipelines has not previously been quantified nor tested explicitly. We developed a $\mu \mathrm{CT}$-based imaging workflow that 
validates neural proteins' durability, neurofilaments, neurotransmitters, and nucleic acids after iodine staining. Our results indicated that a broad array of neural phenotypes were preserved following the iodine stain, imaging, and de-stain process.

Further, we superimposed neuroanatomical datasets to visualize structural diversity for the same individual, successfully demonstrating that brain tissues' suitability for a multimodal visualization pipeline is an important animal model (Fig. 1). DiceCT, therefore, can be used as a readily available, lowcost alternative to $\mu \mathrm{MRI}$ for combined cellular and gross-level imaging modalities in organismal and biomedical neuroscience applications. Below we discuss the implications of our findings, factors that may influence workflow products, and the potential that multiscale, same-sample approaches hold for advancing neuroanatomical research.

\subsection{Effects of diceCT on histology}

Our approach allowed us to survey tissue integrity, which was maintained throughout the workflow's diceCT and histology steps. DiceCT samples undergo formalin fixation, thermal shifts between refrigerated $\left(4^{\circ} \mathrm{C}\right)$, room $\left(25^{\circ} \mathrm{C}\right)$, and CT-scanner cabinet $\left(>25^{\circ} \mathrm{C}\right)$ temperatures, weeks in iodine stain without refrigeration, exposure to high-powered X-rays, and de-staining using alternating baths of sodium thiosulfate and deionized water (Gignac et al. 2016). Exposure to and changes between these environments hold the potential to stress and damage brain tissue beyond its capacity to maintain reactivity to histological stains. Instead, we found that numerous cellular targets remained robust to additional histological stains after implementing diceCT protocols. Tissue sectioning and targetability for immunohistochemistry with fluorescence, fluorescent labeling, and standard acetate-based cytological staining were maintained after diceCT treatment. This was evidenced by visual and quantitative confirmation of sufficient histological labeling following de-staining with sodium thiosulfate. Antiparvalbumin targets calcium-binding proteins and GABAergic neurons, anti-DARPP-32, which targets D1 dopamine receptors, DAPI, which targets nucleic acids; and cresyl violet, which targets RNA, all showed high histological fidelity in experimental and control tissues. The histological staining agents' binding without color pollution from residual iodine illustrates that iodine was adequately removed from brain tissues by the de-staining protocol. The reversibility of diceCT staining is an essential potential differentiator when considering multimodal contrast-enhanced imaging methods (see the survey of contrast agents in Gignac et al. 2016) and a key factor that enables the integrated imaging pipeline presented here. We found it highly valuable to bisect the brains for de-staining, parallel to the plane for histological sectioning, because this provided an additional surface area for sodium thiosulfate and deionized water to diffuse, improving the efficiency of de-staining. By anticipating the plane of the histological section, we minimized unnecessary physical damage to the samples. We recommend similar approaches by other workers undertaking diceCT-histology imaging projects.

Our results show a straightforward pattern of histological robusticity of rodent brain tissues and cellular structures after diceCT. However, we also found that GFAP intensity deviated from the staining mentioned above outcomes. Anti-GFAP densitometry comparisons quantified differences in stain expression during

Page $11 / 25$ 
astrocyte staining, and astrocyte intensities illustrated that experimental brains showed significantly reduced targetability of GFAP (Table 1). Glial fibrillary antigen protein expression is modulated in part by lipopolysaccharides (Kang et al. 2019), which are upregulated during astrocyte proliferation. The affinity of Triiodide for lipids and carbohydrates may contribute to a triiodide-lipopolysaccharide binding that depolymerizes GFAP. Consuming GFAP by this process would reduce available targets for anti-GFAP, also reducing immunostaining intensity. Notably, this phenomenon is likely more pronounced during adolescence, when astrocytogenesis is high (Leal et al. 1997; Akdemir et al. 2020), which characterizes our sample. Thus, the GFAP intensity reduction documented here following diceCT staining may be less pronounced for adult brains. Further evaluation of post-diceCT histology that targets positively charged macromolecules is undoubtedly warranted. Regardless of the mechanism, reduced anti-GFAP values remained sufficient, even in our adolescent sample, for use as a "phenotyping" marker to distinguish cells as belonging to glial versus neuronal populations (Table 1; Fig. 3).

The combination of unaffected anti-parvalbumin, anti-DARPP-32, DAPI, and cresyl violet labeling alongside reduced anti-GFAP labeling leads to a broad and preliminary guideline: cellular targets that are eosinophilic appear more likely to be robust to the diceCT staining and de-staining protocols. Eosinophilic neural proteins, dopamine receptors, and nucleic acids, including those we targeted histologically, have net negative charges (i.e., low isoelectric points; Haiech et al. 1979; Hemmings et al. 1984; Lipfert et al. 2014; O'Dowd et al. 2014). This suggests that they repel the negatively charged Triiodide $\left(I_{3}{ }^{-}\right)$molecules used in diceCT staining. Therefore, Triiodide may fail to bind these substrates, likely contributing to their preservation for multimodal staining pipelines using diceCT. This suggests that histology, especially immunohistochemistry, following diceCT should target substrates that are basic, have low isoelectric points, or are eosinophilic to produce the best quality results.

We found comparable somata diameter measurements between control and experimental tissues, identifying no significant differences in neuronal size in the MPFC, DG, and SNr. This finding indicates that soft-tissue distortions, such as volumetric shrinkage, may occur due to iodine staining (Vickerton et al. 2013; Gignac et al., 2016) not appear to impact neuron size, at least after de-staining and histological preparation. Likewise, comparable amounts of mPFC-labeled cells between control and experimental brains indicate that the addition of diceCT to brain-histology protocols does not impact cell densities. This suggests that any osmotic water loss due to the staining agent's high-salt content during the diceCT phase of the pipeline can be reconstituted during the de-staining process by employing deionized water baths. Thus, the diceCT imaging pipeline we articulate here does not demonstrably alter brain tissue samples' cellular configuration. Taken together, we anticipate that combining diceCT with histological analyses holds enormous promise as a powerful tool for documenting neuroanatomy across spatial scales and in 3D.

\subsection{Considerations for the preservation of nervous tissues}

Nervous tissues must be rapidly fixed with an appropriate concentration and duration of formaldehyde to conduct histological preparations of the brain (Fox et al. 1985; Hughes et al. 2016b). Consequently, not all 
approaches to preserving samples will be compatible with our pipeline, and brain preservation methods should be referenced before imaging attempts. For example, although suitable diceCT images of the brain have been achieved from ethanol-fixed specimens (e.g., Prötzel et al. 2018), formaldehyde fixation of nervous tissues is considered to be optimal for many steps of histology after diceCT. These include tissue sectioning, immunohistochemical staining, and other cell-based stains (Berod et al. 1981). To ensure that the most neuroanatomical data can be gleaned from a single sample, researchers should consider what type of fixative and method of specimen preservation is required to enable continued use of the tissue during downstream investigations. For example, earlier histology efforts by AMK using postdiceCT rat brain samples did not exhibit reactivity when subjected to a wide variety of staining techniques, including immunohistochemistry and cytological-based stains. These prior results were primarily attributed to excessive sample storage post-de-staining in formaldehyde. Therefore, we strongly recommend avoiding over-fixation in order to achieve the best results. Finally, our study's potential limitation is that brain tissues can be distorted from shrinkage due to fixation and over-straining (Gignac and Kley 2018). There are physical methods and mathematical approaches to address these issues (Weisbecker 2012; Taylor et al. 2020); however, some may be incompatible with the multimodal pipeline described here.

\section{Conclusions}

To our knowledge, we are the first to demonstrate that strong cytoplasmic labeling of neurons with cresyl violet staining and immunofluorescence after diceCT brain imaging is preserved. The multiscale examination of precious biological samples enables an advantageous alternative to single-modality pipelines. Multiscale, same-sample approaches provide greater detail for anatomical regions than is possible by using one instrument or method alone. Beyond the conglomerate of staining techniques described here, we also suggest exploring additional essential cellular markers (e.g., tyrosine hydroxylase, choline acetyltransferase) and transcription factors (e.g., c-Fos) following diceCT, may provide fruitful additions to integrated brain-imaging pipelines. We further recommend $\mu \mathrm{CT}$ scanning specimens before any contrast enhancement to capture skeletal morphology, such as the braincase or whole skull. These more traditional $\mu \mathrm{CT}$ datasets can be combined with whole-brain diceCT and region-of-interest histology to study structural anatomy across orders of magnitude in spatial scale that achieves ample coverage of external morphology and internal anatomy-resulting in a cell-to-skull hierarchy of data. Whether harnessed for model organisms (Cole 1944; Preuss 2000; Keifer and Summers 2016; Khan et al. 2018), translational research (Bonnier et al. 2014; Vazquez-Sanroman et al. 2017; Kim et al. 2019), or comparative neuroanatomical studies (Yartsev 2017), pipelines such as these hold great promise for unlocking data that can be unintentionally trapped by single-method preparations, especially those that do not adequately preserve deep internal soft tissues, such as the brain. To unlock general principles of nervous system organization across the tree of life (Butler and Hodos 2005; Manger et al. 2008; Carlson 2012; Striedter et al. 2014; Brenowitz and Zakon 2015; Russell et al. 2017), highly integrative approaches, as we have demonstrated here, are essential (Schwenk et al. 2009; Dowling et al. 2010; Vogelstein et al. 2014). 


\section{Declarations}

\section{Acknowledgments}

We owe special thanks to all UTEP Systems Neuroscience Laboratory members, especially Daniel F. Hughes and Arshad M. Khan, for their valuable comments and feedback. We thank M. Hill and the AMNH Microscopy and Imaging Facility for assistance.

\section{Funding}

PMG was supported by NSF (1450850, 1457180, \& 1754659) and the American Association for Anatomy. DVS, HDO, and PMG were supported by the OSU-CHS department of Anatomy and Cell Biology. DVS and HDO were also supported by the OSU-CHS Office of the Vice President for Research. JS was supported by a pre-doctoral fellowship from the National Mexican Council of Education (CONACyt)

\section{Compliance with ethical standards}

\section{Research involving human participants and animals}

The Oklahoma State University Institutional Animal Care and Use Committee approved all surgical and testing procedures and conformed to NIH guidelines.

\section{Conflict of interest}

The authors declare no conflicts of interest.

\section{References}

Akdemir ES, Huang A Y-S, Deneen B (2020) Astrocytogenesis: where, when, and how [version 1; peer review: 2 approved] F1000Research 2020, 9(F1000 Faculty Rev):233.

https://doi.org/10.12688/f1000research.22405.1

Alonso JR, Covenas R, Lara J, Aijon J (1990) Distribution of parvalbumin immunoreactivity in the rat septal area. Brain Res Bull 24:41-48. https://doi.org/10.1016/0361-9230(90)90287-A

Anderson R, Maga AM (2015) A novel procedure for rapid imaging of adult mouse brains with microCT using iodine-based contrast. PLoS ONE 10:e0142974. https://doi.org/10.1371/journal.pone.0142974

Basser PJ, Mattiello J, LeBihan D (1994) Estimation of the effective self-diffusion tensor form the NMR spin echo. J Mag Res B 103:247-224. https://doi.org/10.1006/jmrb.1994.1037

Berod A, Hartman BK, Pujol JF (1981) Importance of fixation in immunohistochemistry: Use of formaldehyde solutions at variable $\mathrm{pH}$ for the localization of tyrosine hydroxylase. J Histoch \& Cytoch 29:844-850. https://doi.org/10.1177\%2F29.7.6167611 
Bonnier G, Roche A, Romascano D et al (2014) Advanced MRI unravels the nature of tissue alterations in early multiple sclerosis. Ann Clin TransI Neurol 1(6):423-432. https://doi.org/10.1002/acn3.68

Bowler K, Tirri R (1974) The temperature characteristics of synaptic membrane ATPases from immature and adult rat brain. J Neurochem 23:611-613. https://doi.org/10.1111/j.1471-4159.1974.tb06068.x

Brenowitz EA, Zakon HH (2015) Emerging from the bottleneck: Benefits of the comparative approach to modern neuroscience. Trends Neurosci 38:273-278. https://doi.org/10.1016/j.tins.2015.02.008

Burger FJ, Fuhrman FA (1964) Evidence of injury by heat in mammalian tissues. Am J Physiol 206:10571064. https://doi.org/10.1152/ajplegacy.1964.206.5.1057

Burnett TL, McDonald SA, Gholinia A et al (2014) Correlative tomography. Scientific Reports 4:4711. https://doi.org/10.1038/srep04711

Butler A B, Hodos W (2005) Comparative Vertebrate Neuroanatomy: Evolution and Adaptation. John Wiley \& Sons. [ISBN: 978-0-471-73384-3]

Caplan J, Niethammer M, Taylor II RM, Czymmek KJ (2011) The power of correlative microscopy: Multimodal, multi-scale, multi-dimensional. Curr Opin Struc Biol 21:686-693. https://doi.org/10.1016/j.sbi.2011.06.010

Carlson BA (2012) Diversity matters: Diversity Matters: The importance of comparative studies and the potential for synergy between neuroscience and evolutionary biology. Arch Neurol 69: 987-993. https://doi.org/10.1001/archneurol.2012.77

Chen Y, Lin G, Chen Y, Fok A, Slack JM (2012) Micro-computed tomography for visualizing limb skeletal regeneration in young Xenopus frogs. Anat Rec 295:1562-1565. https://doi.org/10.1002/ar.22496

Cole FJ (1944) A History of Comparative Anatomy from Aristotle to the Eighteenth Century. MacMillan \& Co. [ISBN: 9780486602240]

Damadian R, Minkoff L, Goldsmith M, Stanford M, Koutcher J (1976) Field focusing nuclear magnetic resonance (FONAR): visualization of a tumor in a live animal. Science 194:1430-1432. https://doi.org/10.1126/science.1006309

de Crespigny A, Bou-Reslan H., Nishimura MC, Phillips H, Carano RA, D'Arceuil HE (2008) 3D micro-CT imaging of the postmortem brain. J Neurosci Meth 171:207-213.

https://doi.org/10.1016/j.jneumeth.2008.03.006

Ding SL, Royall JJ, Sunkin SM et al (2016) Comprehensive cellular-resolution atlas of the adult human brain. J Comp Neurol 524:3127-3481. https://doi.org/10.1002/cne.24080 
Dodd SJ, Williams M, Suhan JP, Williams DS, Koretsky AP, Ho C (1999) Detection of single mammalian cells by high-resolution magnetic resonance imaging. Biophys J 76:103-109.

https://doi.org/10.1016/S0006-3495(99)77182-1

Dowling AP, Bauchan GR, Ochoa R, Beard JJ (2010) Scanning electron microscopy vouchers and genomic data from an individual specimen: Maximizing the utility of delicate and rare specimens. Acarologia 50:479-485. https://doi.org/10.1051/acarologia/20101983

Feldkamp LA, Goldstein SA, Parfitt AM, Jesion G, Kleerekoper M (1989) The direct examination of threedimensional bone architecture in vitro by computed tomography. J Bone Min Res 4:3-11. https://doi.org/10.1002/jbmr.5650040103

Fox CH, Johnson FB, Whiting J, Roller PP (1985) Formaldehyde fixation. J Histochem Cytochem 33: 845853. https://doi.org/10.1177/33.8.3894502

Gignac PM, Kley NJ (2018) The utility of diceCT imaging for high-throughput comparative neuroanatomical studies. Brain Behav Evol 91:180-190. https://doi.org/10.1159/000485476

Gignac PM, Kley NJ, Clarke JA (2016) Diffusible iodine-based contrast-enhanced computed tomography (diceCT): An emerging tool for rapid, high-resolution, 3-D imaging of metazoan soft tissues. J Anat 228:889-909. https://doi.org/10.1111/joa.12449

Gold S, Cahani M, Sohmer H, Horowitz M, Shahar A (1985) Effects of body temperature elevation on auditory nerve-brain-stem evoked responses and EEGs in rats. Electroencephalogr Clin Neurophysiol 60, 146-153. https://doi.org/10.1016/0013-4694(85)90021-5

Gwóźdź B, Dyduch A, Grzybek H, Panz B (1978). Structural changes in brain mitochondria of mice subjected to hyperthermia. Exp Path Bd 15:124-126. https://doi.org/10.1016/S0014-4908(78)80077-5

Gwóźdź B, Krauze M, Dyduch A (1970) Investigations on oxidative phosphorylation in the brain tissue of animals subjected to high temperature. Acta Physiol Pol 21: 239-245. [PMID: 5420887]

Haiech J, Derancourt J, Pechère J-F, Demaille JG (1979) Magnesium and calcium binding to parvalbumins: evidence for differences between parvalbumins and an explanation of their relaxing function. Biochemistry 19:2752-2758. https://doi.org/10.1021/bi00580a010

Hawkins RA, Hoh C, Glaspy J et al (1992) The role of positron emission tomography in oncology and other whole-body applications. Sem Nucl Med 22:268-284. https://doi.org/10.1016/S00012998(05)80121-7

Heimel P, Swiadek NV, Slezak P, Kerbl M, Schneider C, Nürnberger S, Redl H, Teuschle AH Hercher D (2019) lodine-Enhanced Micro-CT Imaging of Soft Tissue on the Example of Peripheral Nerve Regeneration. Contrast Media Mol Imaging 2019:7483745:1-15. https://doi.org/10.1155/2019/7483745 
Hemmings Jr HC, Greengard P (1986) DARPP-32, a dopamine-regulated phosphoprotein. Prog Brain Res 69:149-159. https://doi.org/0.1016/S0079-6123(08)61056-0

Hemmings Jr HC, Nairn AC, Aswad DW, Greengard P (1984) DARPP-32, a dopamine- and adenosine 3':5'monophosphate-regulated phosphoprotein enriched in dopamine-innervated brain regions. II. Purification and characterization of the phosphoprotein from bovine caudate nucleus. J Neuros 4:99-110.

https://doi.org/10.1523/JNEUROSCI.04-01-00099.1984

Herdina AN, Plenk H, Benda P (2015) Correlative 3D-imaging of Pipistrellus penis micromorphology: Validating quantitative microCT images with undecalcified serial ground section histomorphology. J. Morph 276:695-706. https://doi.org/10.1002/jmor.20372

Hinshaw WS, Bottomley PA, Holland GN (1977) Radiographic thin-section image of the human wrist by nuclear magnetic resonance. Nature 270:722-723. https://doi.org/10.1038/270722a0

Hopkins TM, Heilman AM, Liggett JA, LaSance K, Little KJ, Hom DB, Minteer DM, Marra KG, Pixley SK (2015) Combining micro-computed tomography with histology to analyze biomedical implants for peripheral nerve repair. J Neurosci Meth 255:122-130. https://doi.org/10.1016/j.jneumeth.2015.08.016

Hounsfield GN (1977) The E.M.I. Scanner. Proc Roy Soc Lond B 195:281-289. https://doi.org/10.1098/rspb.1977.0008

Hughes DF, Gignac PM, Greenbaum E, Khan AM (2016a) Documenting brain diversity in field-caught lizards, from skull to cell: Initial development of a processing pipeline for top down, multi-scale structural analyses of a single brain by integrating specialized microcomputed tomography (diceCT), Nissl-based cytoarchitectonics, and immunohistochemistry. Program No. 778.02. 2016 Neuroscience Meeting Planner.

Hughes DF, Walker EM, Gignac PM et al (2016b) Rescuing perishable neuroanatomical information from a threatened biodiversity hotspot: Remote field methods for brain tissue preservation validated by cytoarchitectonic analysis, immunohistochemistry, and x-ray microcomputed tomography. PLoS ONE 11:e0155824. https://doi.org/10.1371/journal.pone.0155824

Jackson JA, Langham WH (1968) Whole-Body NMR Spectrometer. Rev Sci Instrum 39:510-5.13. https://doi.org/10.1063/1.1683420

Jeffery NS, Stephenson RS, Gallagher JA, Jarvis JC, Cox PG (2011) Micro-computed tomography with iodine staining resolves the arrangement of muscle fibres. J Biomech 44:189-192.

https://doi.org/10.1016/j.jbiomech.2010.08.027

Jellison BJ, Field AS, Medow J, et al (2004) Diffusion tensor imaging of cerebral white matter: a pictorial review of physics, fiber tract anatomy, and tumor imaging patterns. Am J Neuroradiol 25:356-369.

[PMID: 15037456] 
Jones T, Townsend D (2017) History and future technical innovation in positron emission tomography. J Med Imaging 4:011013. https://doi.org/10.1117/1.JMI.4.1.011013

Kang J-B, Park D-J, Shah M-A, Kim M-O, Koh P-O (2019) Lipopolysaccharide induces neuroglia activation and NF-kB activation in cerebral cortex of adult mice. Lab Anim Res 35:19.

https://doi.org/10.1186/s42826-019-0018-9

Karreman MA, Mercier L, Schieber NL et al (2016) Fast and precise targeting of single tumor cells in vivo by multimodal correlative microscopy. J Cell Sci 129:444-456. https://doi.org/10.1242/jcs.181842

Keifer J, Summers CH (2016) Putting the "biology" back into "neurobiology": The strength of diversity in animal model systems for neuroscience research. Front Sys Neurosci 10:69. https://doi.org/10.3389/fnsys.2016.00069

Khan AM, Grant AH, Martinez A et al (2018) Mapping molecular datasets back to the brain regions they are extracted from: Remembering the native countries of hypothalamic expatriates and refugees. Adv Neurobiol 21:101-193. https://doi.org/10.1007/978-3-319-94593-4_6

Kim C-B, Park S-J, Jeong J-C (2019) Construction of 3D-rendering imaging of an ischemic rat brain model using the planar FMMD technique. Sci Rep 9:19050. https://doi.org/10.1038/s41598-019-55585-x

Kondo H, Fukuda H, Ono H et al (2001) Sodium thiosulfate solution spray for relief of irritation caused by Lugol's stain in chromoendoscopy. Gastrointest Endosc 53:199-202.

https://doi.org/10.1067/mge.2001.110730

Leal RB, Gonçalves CA, Rodnight R (1997) Calcium-dependent phosphorylation of glial fibrillary acid protein (GFAP) in the rat hippocampus: a comparison of the kinase/phosphatase balance in immature and mature slices using tryptic phosphopeptide mapping. Dev Brain Res 105:1-10.

https://doi.org/10.1016/s0165-3806(97)00113-2

Lautenschlager S, Bright JA, Rayfield EJ (2014) Digital dissection-using contrast-enhanced computed tomography scanning to elucidate hard-and soft-tissue anatomy in the Common Buzzard Buteo buteo. $\mathrm{J}$ Anat 224:412-431. https://doi.org/10.1111/joa.12153

Lauterbur PC (1974) Magnetic resonance zeugmatography. Pure Appl Chem 40:119-157. https://doi.org/10.1351/pac197440010149

Lipfert J, Doniach S, Das R, Herschlag D (2014) Understanding nucleic acid-ion interactions. Annu Rev Biochem 83:813-841. https://doi.org/10.1146/annurev-biochem-060409-092720

Manger P, Cort J, Ebrahim N, Goodman A, Henning J, Karolia M, Rodrigues SL, Strkalj G (2008) Is 21st century neuroscience too focused on the rat/mouse model of brain function and dysfunction? Front Neuroanat 2:5. https://doi.org/10.3389/neuro.05.005.2008 
McDonald LW, Hayes TL (1969) Correlation of scanning electron microscope and light microscope images of individual cells in human blood and blood clots. Exp Mol Pathol 10:186-198.

Millan N, Murdock LL, Bleier R, Siegel FL (1979) Effects of the acute hyperthermia on polyribosomes, in vivo protein synthesis and ornithine decarboxylase activity in the neonatal rat brain. $J$ Neurochem 32:311-317.

Mikula, S., Trotts, I., Stone, J., and Jones, E.G. 2007. Internet-Enabled High-Resolution Brain Mapping and Virtual Microscopy. Neurolmage. 35(1):9-15

Morales AG, Stempinski ES, Xiao X, Patel A, Panna A, Olivier KN, McShane PJ, Robinson C, George AJ, Donahue DR, Chen P (2016) Micro-CT scouting for transmission electron microscopy of human tissue specimens. J of Microsc 263:113-117.

Muehllehner G, Buchin MP, Dudek JH (1976) Performance parameters of a positron imaging camera. IEEE T Nucl Sci 23:528-537. https://doi.org/10.1109/TNS.1976.4328300

Naumann A, Olsson L (2018) Three-dimensional reconstruction of the cranial and anterior spinal nerves in early tadpoles of Xenopus laevis (Pipidae, Anura). J Comp Neurol 526:836-857.

O'Dowd BF, Ji X, Nguyen T, George S R (2012) Two amino acids in each of $D_{1}$ and $D_{2}$ dopamine receptor cytoplasmic regions are involved in D1-D2 heteromer formation. Biochem Biophys Res Commun 417(1):23-28. https://doi.org/10.1016/j.bbrc.2011.11.027

Parlanti P, Cappello V, Brun F, Tromba G, Rigolio R, Tonazzini I, Cecchini M, Piazza V, Gemmi M (2017) Size and specimen-dependent strategy for $\mathrm{x}$-ray micro-CT and TEM correlative analysis of nervous system samples. Sci Rep 7:2858.

Paxinos G, Watson C (2003) The Rat Brain in Stereotaxic Coordinates, 7th edition. Elsevier. [ISBN: 9780123919496]

Pelc NJ (2014). Recent and future directions in CT imaging. Ann Biomed Eng 42(2):260-268. https://doi.org/10.1007/s10439-014-0974-z

Phelps ME, Hoffman EJ, Mullani NA, Higgins CS, Pogossian MMT (1976) Design considerations for a positron emission transaxial tomograph (PETT III). IEEE Trans Nuc Sci 23:516-522.

https://doi.org/10.1109/TNS.1976.4328298

Preuss TM (2000) Taking the measure of diversity: Comparative alternatives to the model-animal paradigm in cortical neuroscience. Brain, Behavior and Evolution 55:287-299.

Prötzel D, Vences M, Hawlitschek O, Scherz MD, Ratsoavina FM, Glaw F (2018) Endangered beauties: Micro-CT cranial osteology, molecular genetics and external morphology reveal three new species of 
chameleons in the Calumma boettgeri complex (Squamata: Chamaeleonidae). Zool J Linn Soc 184:471498.

Rahati M, Nozari M, Eslami H, Shabani M, Basiri M (2016) Effects of enriched environment on alterations in the prefrontal cortex GFAP- and S100B-immunopositive astrocytes and behavioral deficits in MK-801treated rats. Neuroscience 326:105-116. https://doi.org/10.1016/j.neuroscience.2016.03.065

Rüegsegger P, Koller B, Müller R (1996) A microtomographic system for the nondestructive evaluation of bone architecture. Calcified Tissue Int 58:24-29. https://doi.org/10.1007/bf02509542

Russell JJ, Theriot JA, Sood P, Marshall WF, Landweber LF, Fritz-Laylin L, Polka JK, Oliferenko S, Gerbich T, Gladfelter A, Umen, J (2017) Non-model model organisms. BMC Biol 15:55.

Schwenk K, Padilla DK, Bakken GS, Full RJ (2009) Grand challenges in organismal biology. Integr Comp Biol 49:7-14. https://doi.org/10.1093/icb/icp034.

Sengle G, Tufa SF, Sakai LY, Zulliger MA, Keene DR (2013) A correlative method for imaging identical regions of samples by micro-CT, light microscopy, and electron microscopy: Imaging adipose tissue in a model system. J Histochem Cytochem 61:263-271.

Shakhashiri B (1983) Chemical Demonstrations: A Handbook for Teachers of Chemistry, Vol. 3. Madison: University of Wisconsin Press.

Shami GJ, Cheng D, Braet F (2017) Combined multidimensional microscopy as a histopathology imaging tool. J Cell Physiol 232:249-256.

Smith BR, Linney E, Huff DS, Johnson GA (1996) Magnetic resonance microscopy of embryos. Comput Med Imag Grap. 20:483-490. https://doi.org/10.1016/S0895-6111(96)00046-8

Striedter GF et al (2014) NSF workshop report: Discovering general principles of nervous system organization by comparing brain maps across species. J Comp Neurol 522:1445-1453.

Swanson, LW (2018) Brain maps 4.0-Structure of the rat brain: An open access atlas with global nervous system nomenclature ontology and flatmaps. J Comp Neurol 526:935-943.

Taylor MS, O'Brien HD, Gignac PM (2020). Shrinkage after swimming in iodine? Evaluating the use of hydrogel stabilization for reinforcing nervous tissues before iodine diffusion. Society for Integrative and Comparative Biology Abstracts 122-2.

Ter-Pogossian MM, Phelps ME, Hoffman EJ, Mullani NA (1975) A Positron-emission transaxial tomography for nuclear imaging (PETT). Radiology 114:89-98. https://doi.org/10.1148/114.1.89

Trevorrow V, Fashena G (1935) The determination of iodine in biological material. J Biol Chem 110:2938. 
Vazquez-Sanroman DB, Monje RD, Bardo MT (2017) Nicotine self-administration remodels perineuronal nets in ventral tegmental area and orbitofrontal cortex in adult male rats. Addict Biol 22:1743-1755.

Vickerton P, Jarvis J, Jeffery N (2013) Concentration-dependent specimen shrinkage in iodine-enhanced microCT. J Anat 223:185-193. http://doi.org/10.1111/joa.12068

Voie AH, Burns DH, Spelman FA (1993) Orthogonal-plane fluorescence optical sectioning: Threedimensional imaging of macroscope biological specimens. J Microsc 170:229-236.

https://doi.org/10.1111/j.1365-2818.1993.tb03346.x

Vogelstein JT, Park Y, Ohyama T, Kerr RA, Truman JW, Priebe CE, Zlatic M (2014) Discovery of brainwide neural-behavioral maps via multiscale unsupervised structure learning. Science 344:386-392.

Weisbecker V (2012) Distortion in formalin-fixed brains: Using geometric morphometrics to quantify the worst-case scenario in mice. Brain Struct Funct 217:677-685.

Wong MD, Spring S, Henkelman RM (2013) Structural stabilization of tissue for embryo phenotyping using micro-CT with iodine staining. PLoS ONE 8:e84321.

Yartsev MM (2017) The emperor's new wardrobe: Rebalancing diversity of animal models in neuroscience research. Science 358:466-469.

\section{Table}

Table 1. Significance values for ANOVA and $t$-tests of control and post-diceCT samples stained using anti-Parvalbumin, anti-GFAP, DAPI, anti-DARPP-32, and cresyl violet with target neural phenotype indicated. Significance $(\alpha<0.05$; indicated by asterisk) was identified for anti-GFAP densitometry measurements only. Somata diameters, cell counts, and other fluorescent intensity comparisons were not significantly different.

\begin{tabular}{|l|l|l|}
\hline Histological Prep & \multicolumn{1}{|c|}{ Neural Phenotype } & \multicolumn{1}{c|}{ Significance } \\
\hline anti-Parvalbumin & GABAergic interneurons & mPFC: $\mathrm{p}=0.89 ; \mathrm{DG}: \mathrm{p}=0.92$ \\
\hline anti-GFAP & Glial cell intermediate filament proteins & MM: $\mathrm{p}<0.01^{*}$ \\
\hline DAPI & Nucleic acids & mPFC: $\mathrm{p}=0.96$ \\
\hline anti-DARPP-32 & Phosphoprotein in D1 receptor neurons & SNr: $\mathrm{p}=0.41$ \\
\hline cresyl violet & RNA & DG: $\mathrm{p}=0.66$ \\
\hline
\end{tabular}

\section{Figures}




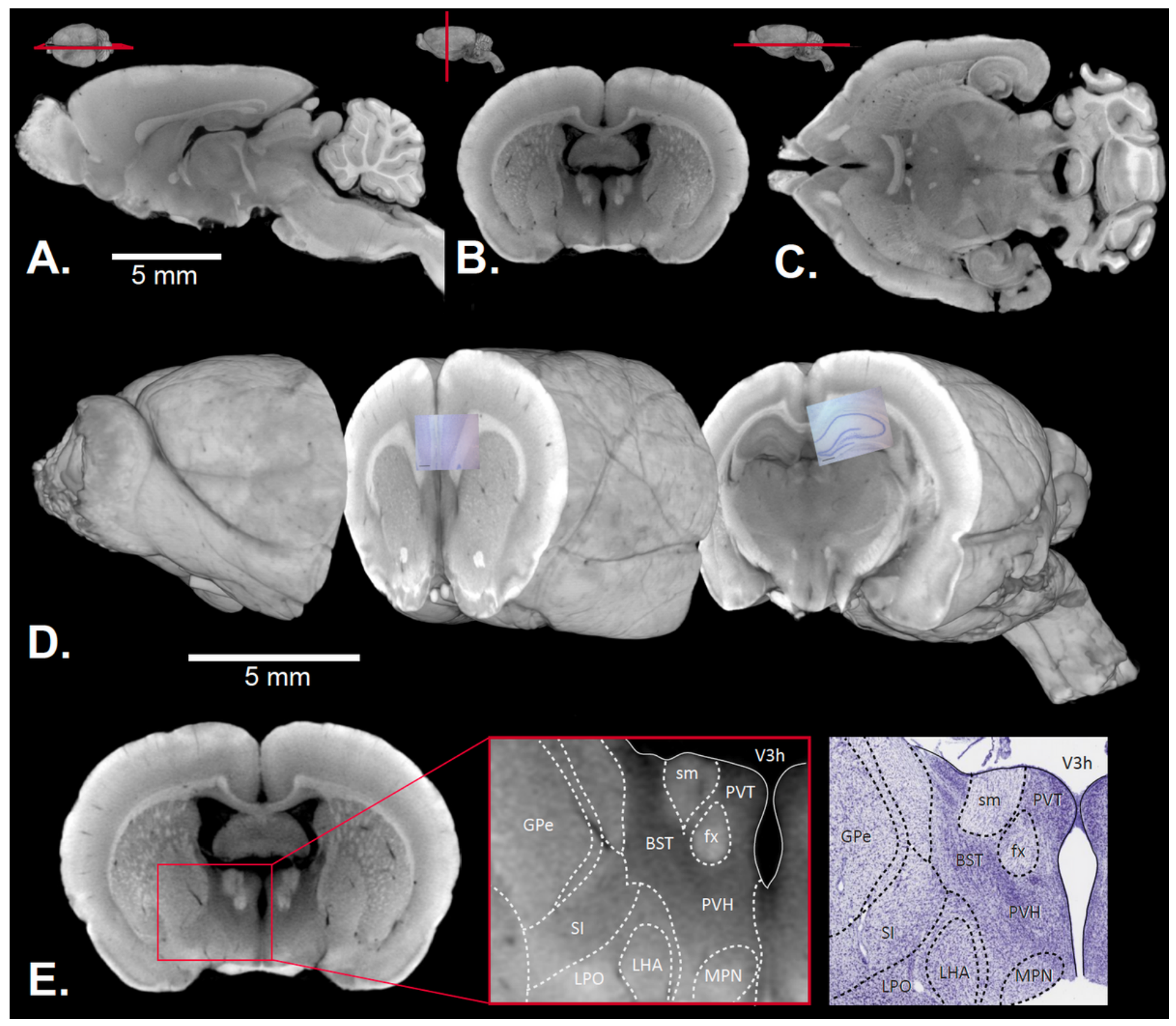

\section{Figure 1}

2D images of an adolescent rat brain imaged using diceCT in (A) parasagittal, (B, E) coronal, and (C) transverse views alongside a (D) 3D projection that has been digitally dissected to show the MPFC and DG. In (D), 2D cresyl violet histological images from the mPFC and DG overlay their positions in the 3D model to illustrate the integration of multiscale bio-imaging approaches. Neuroanatomical comparison (E) of diceCT (left) and cresyl violet histology (right) with regions of interest labeled. Cresyl violet image downloaded from BrainMaps.org (Mikula et al., 2007). Abbreviations: BST, bed nuclei of stria terminalis [Johnston]; $f x$, column of the fornix; GPe, globus pallidus; LHA, lateral hypothalamic area; LPO, lateral preoptic area; MPN, medial preoptic nucleus; PVH, paraventricular nucleus hypothalamus; PVT, paraventricular nucleus thalamus; SI, substantia innominate [Reil, Reichert]; sm, stria medullaris; and V3h, third ventricle. Nomenclature following Swanson, 2018 
Figure 2

Medial Prefrontal Cortex

Saline-PFH-Perfusion and Post-diceCT
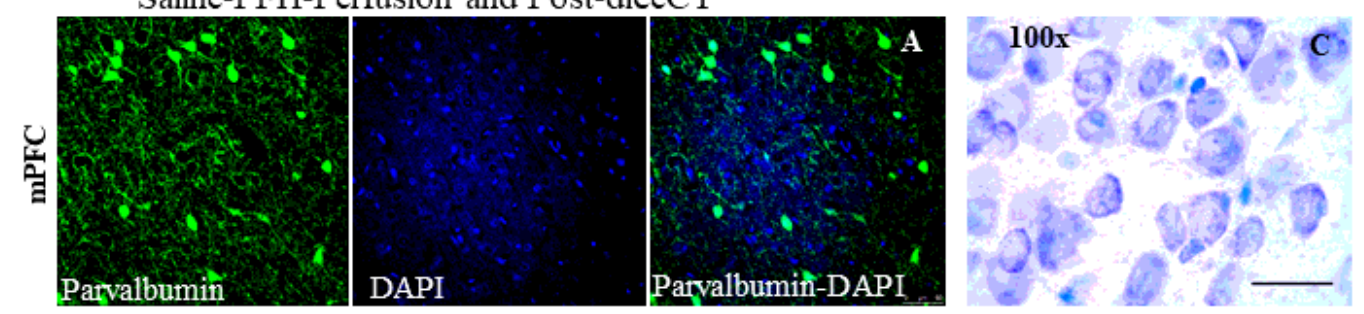

Saline-PFH-Perfusion (Control)
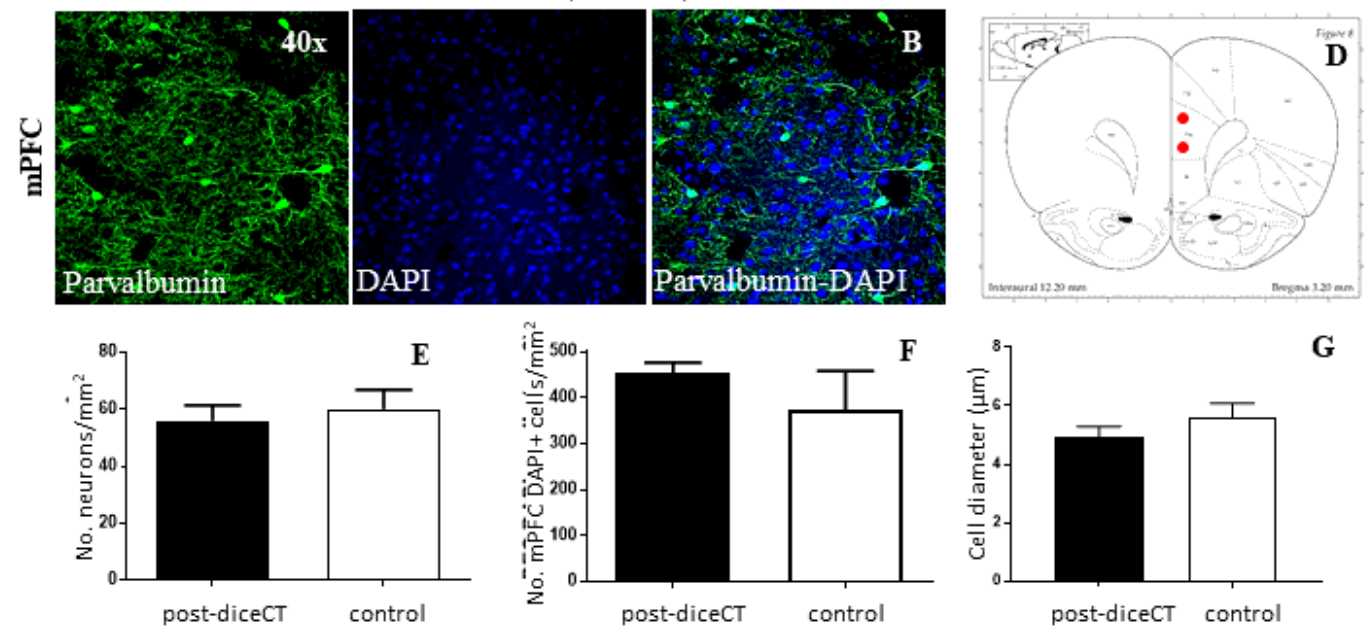

Medial Mammillary nucleus
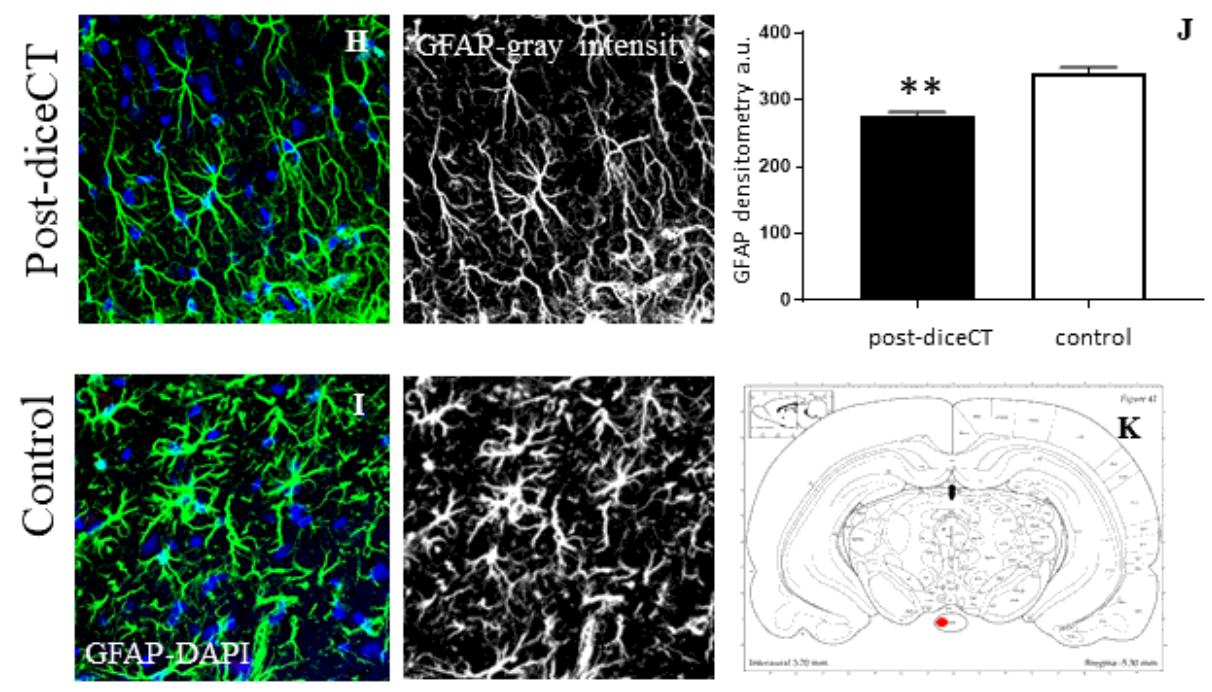

\section{Figure 2}

(A, B) Immunostained and (C) cresyl violet stained mPFC (scale bar is $20 \mu \mathrm{m}$ ). PRV immunoreactivity (green), DAPI labeling (blue), and their co-visualization (merge) in mPFC from (A) postdiceCT and (B) control subjects; (D) sampled mPFC areas (red) [Paxinos and Watson, 2003]. (E) Mean $( \pm S E M)$ number of PRV immunoreactive cells for post-diceCT and control mPFC per mm2. (F) Mean 
( \pm SEM) number of DAPI-positive cells for post-diceCT and control mPFC per mm2. (G) Mean ( \pm SEM) cell diameter of DAPI-positive cells for post-diceCT and control mPFC.

\section{Figure 3}

\section{Hippocampus}

Nissl Staining: Saline-PFH-Perfusion and Post-diceCT
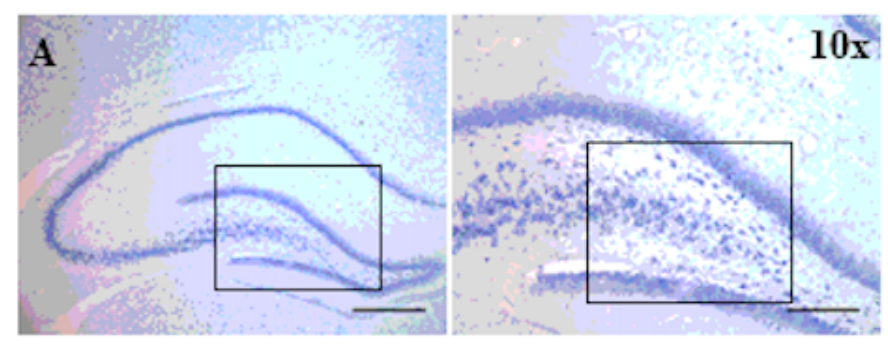

diceCT

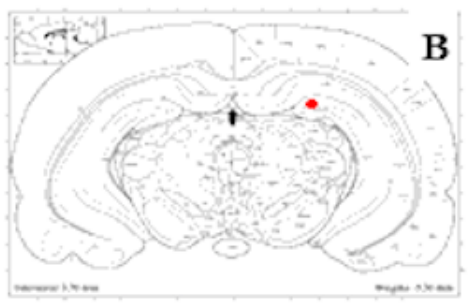

B
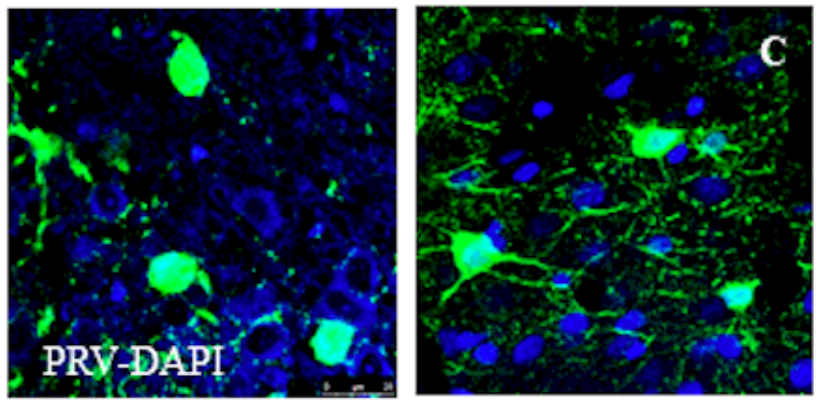

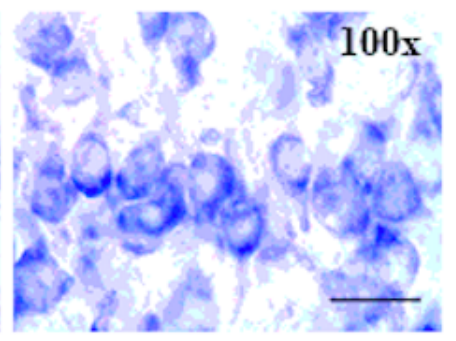

Control

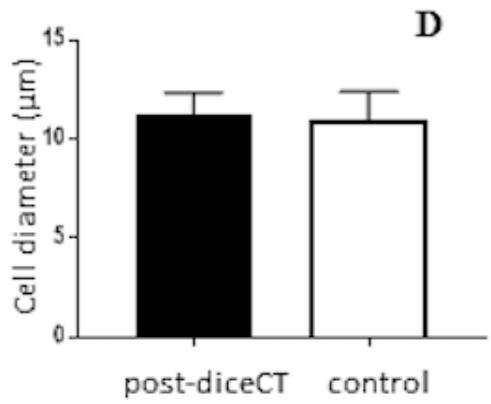

Sustantia Nigra
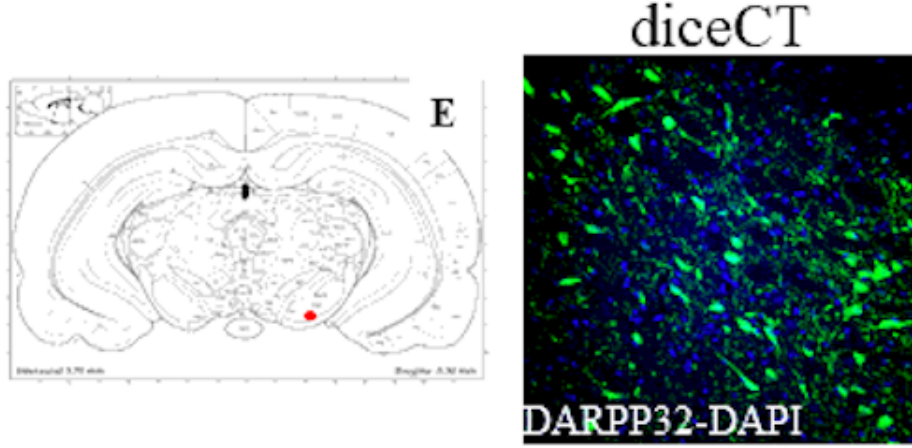

Control
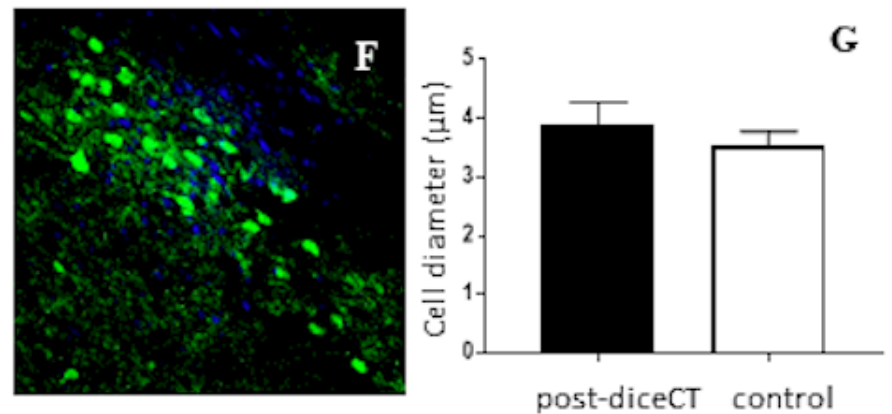

\section{Figure 3}

GFAP-immunoreactive (green) and DAPI-positive (blue) cells in (A) post-diceCT and (B) and control MM. Scale bar is $20 \mu \mathrm{m}$. (C) Mean ( \pm SEM) attenuation units (a.u.) of GFAP-positive cells for post-diceCT and control MM; (D) sampled MM area (red) [Paxinos and Watson, 2003]. 


\section{Image not available with this version}

\section{Figure 4}

(A) cresyl violet-stained DG, insets mark regions magnified (4-100x; scale bars: 600, 250, 60, $25 \mu \mathrm{m}$ ) in successive panels from left to right. (B) Sampled DG area (red) [Paxinos and Watson, 2003]. (C) PRV immunoreactivity (green) and DAPI-positive (blue) cells in post-diceCT and control DG. (D) Mean ( \pm SEM) cell diameter of DAPI-positive cells for post-diceCT and control DG.

\section{Image not available with this version}

Figure 5

(A) Sampled area a SNr (red) [Paxinos and Watson, 2003]; (B) DARPP-32-immunoreactive (green) and DAPI-positive (blue) cells in post-diceCT and control SN. (C) Mean ( \pm SEM) cell diameter of DAPI-positive cells for post-diceCT and control SNr. 